\title{
PAPER Structure Learning of Bayesian Networks Using Dual Genetic Algorithm*
}

\author{
Jaehun LEE $^{\dagger}$, Wooyong CHUNG ${ }^{\dagger}$, Nonmembers, and Euntai $\mathrm{KIM}^{\dagger \dagger \mathrm{a})}$, Member
}

\begin{abstract}
SUMMARY A new structure learning approach for Bayesian networks (BNs) based on dual genetic algorithm (DGA) is proposed in this paper. An individual of the population is represented as a dual chromosome composed of two chromosomes. The first chromosome represents the ordering among the $\mathrm{BN}$ nodes and the second represents the conditional dependencies among the ordered $\mathrm{BN}$ nodes. It is rigorously shown that there is no $\mathrm{BN}$ structure that cannot be encoded by the proposed dual genetic encoding and the proposed encoding explores the entire solution space of the BN structures. In contrast with existing GA-based structure learning methods, the proposed method learns not only the topology of the BN nodes, but also the ordering among the BN nodes, thereby, exploring the wider solution space of a given problem than the existing method. The dual genetic operators are closed in the set of the admissible individuals. The proposed method is applied to real-world and benchmark applications, while its effectiveness is demonstrated through computer simulation.

key words: Bayesian network, genetic algorithms, structure learning, dual chromosomes
\end{abstract}

\section{Introduction}

The Bayesian network (BN) is one of the most popular formalisms for reasoning under uncertainty in Artificial Intelligence (AI). The BN is a graphical model that denotes joint probabilistic distribution among variables of interest based on the variables' probabilistic relationships. The structure of the $\mathrm{BN}$ is represented as a directed acyclic graph (DAG). In a DAG, each node represents a variable that takes on a value over both continuous and discrete data set of domain and is connected with its parent's nodes. Each arc represents the conditional dependency between the two nodes so connected. With the development of large-scale database systems, the BN has become a popular knowledge representational scheme for probabilistic knowledge in data mining and knowledge discovery [1]-[3].

In building the $\mathrm{BN}$, which represents the conditional dependencies in a database of cases, the problem of searching for the structure of the $\mathrm{BN}$ is both important and difficult. A large number of possible DAG structures could be determined from a small number of nodal connections. To

Manuscript received July 27, 2006.

Manuscript revised June 25, 2007.

${ }^{\dagger}$ The authors are with the CILAB, School of Electrical and Electronic Engineering, Yonsei University, 134, Shinchon-Dong, Sudaemun-ku, Seoul 120-749, Korea.

${ }^{\dagger \dagger}$ The author is with the School of Electrical and Electronic Engineering, Yonsei University, 134, Shinchon-Dong, Sudaemun-ku, Seoul 120-749, Korea.

${ }^{*}$ This work was supported by the Ministry of Commerce, Industry and Energy of Korea (HISP).

a)E-mail: etkim@yonsei.ac.kr

DOI: 10.1093/ietisy/e91-d.1.32 solve this problem, several methods have been reported [4][10] for the structure learning of the BN. The most popular method for $\mathrm{BN}$ structure learning from a database is the $\mathrm{K} 2$ algorithm [10]. The K2 algorithm assumes that an ordering on the variables is given and searches for an appropriate structure heuristically. But this method is heuristic and does not guarantee that an optimal structure will be found.

On the other hand, GAs have been successfully applied to various optimization problems in real world applications. They are well suited to the exploration of complex search spaces. As an alternative scheme to K2, the learning methods based on the GAs have been reported [7]-[9], [11], [12]. The GA, however, is not a ready made optimization tool for $\mathrm{BN}$ structure learning. The plain GA cannot be applied to the structure learning of $\mathrm{BN}$ and there is no general way to find the optimal structure in the entire DAG space because the plain genetic operations are not closed in the set of the possible BN structures. Thus, in the existing methods, the GAs also restrict the ordering on the variables, in which initially a search is conducted for the solution in the directed graph space, including cyclic and acyclic, and then the cyclic solutions are removed.

In this paper, a new structure learning method of the BNs based on a dual genetic algorithm (DGA) is proposed. In the proposed method, a BN structure is represented as paired chromosomes. One represents the ordering among the BN nodes and the other represents conditional dependencies among the ordered $\mathrm{BN}$ nodes. In contrast with the existing GA-based structure learning methods of the $\mathrm{BN}$ [7], the proposed method learns not only the topologies of the BN nodes, but also the ordering among the BN nodes, thereby, exploring the entire solution space of a given problem. The proposed method is somewhat similar to [18] but the contribution of this paper is twofold: (1) It is rigorously shown that the dual chromosome can represent all the possible BN structures, thereby exploring the entire $\mathrm{BN}$ structure space and (2) the proposed method uses slightly different genetic operators from [18].

This paper is organized as follows. In Sect. 2, a brief introduction to BNs and GAs is given. In Sect. 3, the DGA for structure learning of the $\mathrm{BN}$ is proposed and the new genetic operations are introduced. In Sect. 4, the proposed method is applied to real-world and benchmark problems: a database of virtual home network systems [13], a database of car diagnosis problems as suggested by Norsys [14], and a database of ALARM networks constructed by Beinlinch et al. [10]. Finally, some conclusions are drawn in Sect. 5. 


\section{Preliminary Fundamentals: Bayesian Networks and Genetic Algorithms}

\subsection{Bayesian Network}

The Bayesian network and associated schemes constitute a probabilistic framework for reasoning under uncertainty and in recent years have gained popularity in the community of artificial intelligence. The BN is composed of a network structure and a set of parameters associated with the structure. The structure of the $\mathrm{BN}$ is a directed acyclic graph (DAG), in which each node represents a random variable that can take on a finite set of values and each arc represents the conditional dependency (topology) between the two nodes [15]. In this paper, we assume that each node takes only discrete values.

To specify the BN completely in conjunction with the DAG, the user must give prior probabilities for all root nodes (nodes with no predecessors) and conditional probabilities for all the other nodes. The joint probability of any particular instantiation of all $n$ variables in the BN can be calculated with

$$
P(U)=\prod_{i} P\left(A_{i} \mid p a\left(A_{i}\right)\right)
$$

where $U=A_{1}, A_{2}, \cdots, A_{n}$ represents $n$ nodes of the $\mathrm{BN}$ and $p a\left(A_{i}\right)$ is the parent set of the variable $A_{i}$. For example, the probability of the BN shown in Fig. 1 is computed by:

$$
\begin{aligned}
P(U)= & P\left(A_{1}\right) P\left(A_{2}\right) P\left(A_{3}\right) P\left(A_{4} \mid A_{1}, A_{2}\right) \\
& \cdot P\left(A_{5} \mid A_{2}, A_{3}\right) P\left(A_{6} \mid A_{4}\right) P\left(A_{7} \mid A_{5}\right)
\end{aligned}
$$

The process of building the $\mathrm{BN}$ can be separated into two tasks: structure learning and parameter learning. Structure learning is a search for an appropriate structure for the $\mathrm{BN}$ such that the BN accommodates the given set of samples. Parameter learning is computation of the conditional probabilities for the given BN structure such that the output of the BN approximates the distribution of the given set of samples. The most popular parameter learning method is the expectation maximization (EM) algorithm [16]. In this paper, the focus is structure learning of the $\mathrm{BN}$ and building an appropriate BN structure such that the BN structure accommodates the given set of samples.

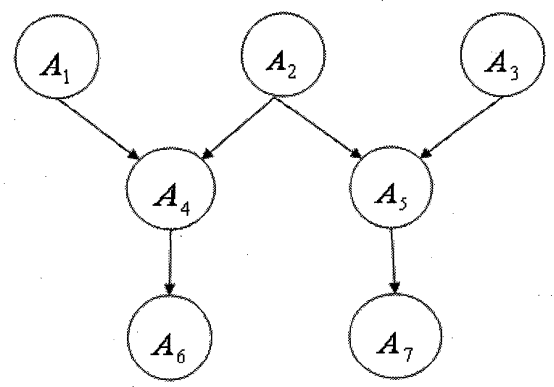

Fig. 1 An example of a Bayesian network.

\subsection{Genetic Algorithms}

The genetic algorithm (GA) is the implementation of a biological metaphor. In the algorithm, learning is viewed as a competition among a population of evolving individuals. The fitness, or goodness, of each individual is evaluated and, according to the computed fitness value, it is determined which one will survive in the next generation. Through operations analogous to gene transfer in sexual reproduction, a new population of candidate solutions is created [17]. The general procedure for GA is summarized as follows. An initial population of individuals called chromosomes is created by random selection and the goodness of each individual is evaluated by a fitness function. Then, a new population of reproduced individuals is selected according to the fitness values. In the selection, the individuals with high fitness values are more likely to reproduce offspring than those with low fitness values. Finally, the selected individuals are mated with others and sometimes mutated. If the new population includes an individual that produces a satisfactory performance or fitness level, then the algorithm stops and the problem is solved. If not, then the algorithm is repeated until a termination condition is satisfied. The pseudo code of a simple genetic algorithm (SGA) is shown below:

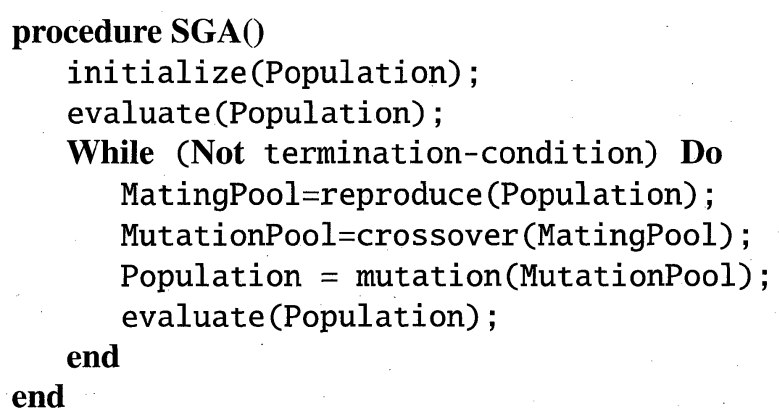

\section{Structure Learning of Bayesian Networks Based on the Dual Genetic Algorithm}

In this section, a new approach for structure learning of BNs based on the dual genetic algorithm is proposed. The proposed method explores a wider solution space than the existing GA-based structure learning method.

\subsection{The Conventional Method for BN Structure Learning}

In this subsection, the conventional method that uses the GA for the structure learning of the BN is explained briefly [7]. The $\mathrm{BN}$ structure with $n$ variables is represented by an $n \times n$ connectivity matrix $C=\left(c_{i, j}\right)$, where

$$
c_{i, j}= \begin{cases}1 & \text { if } i \text { is a parent of } j \\ 0 & \text { otherwise }\end{cases}
$$

and each individual of the population is encoded as a chromosome, 


$$
c_{1,1} c_{1,2} \cdots c_{1, n} c_{2,1} c_{2,2} \cdots c_{2, n} \cdots c_{n, 1} c_{n, 2} \cdots c_{n, n}
$$

With this representation, the plain crossover and mutation operators would produce illegal BN structures. In the previous method, to overcome this problem, the connectivity matrix was assumed to be upper triangular and the connectivity matrix,

$$
\left(\begin{array}{cccccc}
0 & c_{1,2} & c_{1,3} & \cdots & c_{1, n-1} & c_{1, n} \\
0 & 0 & c_{2,3} & \cdots & c_{2, n-1} & c_{2, n} \\
\vdots & \vdots & \vdots & \ddots & \vdots & \vdots \\
0 & 0 & 0 & \cdots & 0 & c_{n-1, n} \\
0 & 0 & 0 & \cdots & 0 & 0
\end{array}\right)
$$

was encoded as a chromosome,

$$
X=c_{1,2} \cdots c_{1, n} c_{2,3} \cdots c_{2, n} \cdots c_{n-2, n-1} c_{n-2, n} c_{n-1, n}
$$

In other words, the ordering among the variables of the BN was fixed and a node $A_{i}$ was allowed to have another node $A_{j}$ as a parent node only if the node $A_{j}$ comes before the node $A_{i}$ in the ordering. This scheme restricts the values of $c_{i, j}(i \geq j)$ to 0 and narrows the search space to the extent that the GA will find only a suboptimal solution, if the fixed ordering of the nodes is wrong.

\subsection{Dual Genetic Algorithm for BN Structure Learning}

The conventional method has the limitation that the ordering between the nodes is predetermined and the nodal connections are upper triangular. The conventional genetic encoding cannot represent all the possible BN structures and the search space was not fully explored to determine the fittest structure of BNs. If there is no prior knowledge about the ordering between variables, which is usually the case, the resulting structure will not be satisfactory.

This paper describes a proposed new genetic method that can be applied to the structure learning of the BNs. In the proposed method, the connectivity matrix is assumed to be upper triangular, but it is not exactly the same as that of the conventional method. The ordering of the nodes is not fixed and it is also learned by the GA. Thus, the entire solution space is searched for the fittest structure of the BN.

\subsubsection{Encoding}

In order to encode all of the information related to the structure learning of the $\mathrm{BN}$ into a chromosome and search the entire solution space for the fittest structure, a dual chromosome is proposed, which is composed of an ordering chromosome and a connectivity chromosome: The ordering chromosome is integer-coded and denotes the ordering among the BN nodes. The connectivity chromosome is binarycoded and denotes the upper triangular connectivity matrix, which represents the connection among the nodes with respect to the ordering chromosome. The ordering chromosome $X_{o}$ and the connectivity chromosome $X_{c}$ are encoded as follows:

$$
\begin{aligned}
& X_{o}=x_{1} x_{2} \cdots x_{n} \\
& X_{c}=c_{1,2} \cdots c_{1, n} c_{2,3} \cdots c_{2, n} \cdots c_{n-2, n-1} c_{n-2, n} c_{n-1, n}
\end{aligned}
$$

where

$$
\begin{aligned}
& x_{i} \text { is an integer and } x_{i} \in\{1, \cdots, n\}, x_{i} \neq x_{j}, \text { iff } i \neq j \\
& C=\left(\begin{array}{cccccc}
0 & c_{1,2} & c_{1,3} & \cdots & c_{1, n-1} & c_{1, n} \\
0 & 0 & c_{2,3} & \cdots & c_{2, n-1} & c_{2, n} \\
\vdots & \vdots & \vdots & \ddots & \vdots & \vdots \\
0 & 0 & 0 & \cdots & 0 & c_{n-1, n} \\
0 & 0 & 0 & \cdots & 0 & 0
\end{array}\right) \\
& c_{i, j}= \begin{cases}1 & \text { if } x_{i} \text { is a parent of } x_{j} \\
0 & \text { otherwise }\end{cases}
\end{aligned}
$$

The ordering chromosome consists of the indices of the variables. If there are $n$ root nodes (nodes without parents) and they have $m$ children nodes, the first $n$ genes of this chromosome are the indices of the root nodes. The next $m$ genes are the indices of nodes which can be the children of the root nodes. This structure is repeated until all of the variables are visited. The connectivity chromosome denotes the connectivity matrix, which defines the dependency relation among the variables. For the evolution of the connectivity chromosome, the plain GA operators are applied. For the evolution of the ordering chromosome, however, the plain GA operators do not work and some genetic operators used in traveling salesman problem (TSP) are applied to find the best ordering of the variables [17]. In [18], several GA operators used in TSP were applied under the $\mathrm{K} 2$ algorithm and it was shown that their performances are almost the same. In the proposed method, the GA operators similar to those of [18] are used. The following examples show the basic idea of the suggested dual genetic encoding. The GA operators for the dual genetic encoding will be explained in detail in the subsequent section.

Example 1 (Encoding of the dual chromosome): Consider two BN structures as shown in Fig. 2.

Each BN is represented by a dual chromosome $\left(X_{i o}, X_{i c}\right)$, in which $i=1,2$ and the subscripts $o$ and $c$ denote an ordering chromosome and a connectivity chromosome, respectively. These chromosomes are explicitly shown in (9).

$$
\begin{aligned}
& X_{1 o}=126354 \\
& X_{1 c}=110000100010010 \\
& X_{2 o}=543126
\end{aligned}
$$
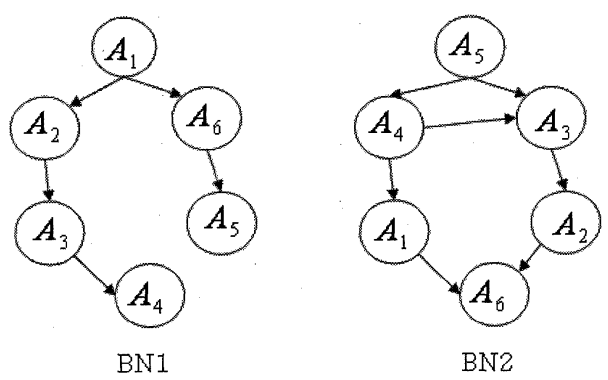

Fig. 2 Two BNs (Example 1). 


$$
X_{2 c}=110001100010011
$$

$C_{i}$ and $\bar{C}_{i}$ denote the connectivity matrices, as shown in (10), created by the proposed dual chromosome $\left(X_{i o}, X_{i c}\right)$ and by the conventional method [7], respectively.

$$
\begin{aligned}
& C_{1}=\left(\begin{array}{llllll}
0 & 1 & 1 & 0 & 0 & 0 \\
0 & 0 & 0 & 1 & 0 & 0 \\
0 & 0 & 0 & 0 & 1 & 0 \\
0 & 0 & 0 & 0 & 0 & 1 \\
0 & 0 & 0 & 0 & 0 & 0 \\
0 & 0 & 0 & 0 & 0 & 0
\end{array}\right), \bar{C}_{1}=\left(\begin{array}{llllll}
0 & 1 & 0 & 0 & 0 & 1 \\
0 & 0 & 1 & 0 & 0 & 0 \\
0 & 0 & 0 & 1 & 0 & 0 \\
0 & 0 & 0 & 0 & 0 & 0 \\
0 & 0 & 0 & 0 & 0 & 0 \\
0 & 0 & 0 & 0 & 1 & 0
\end{array}\right) \\
& C_{2}=\left(\begin{array}{llllll}
0 & 1 & 1 & 0 & 0 & 0 \\
0 & 0 & 1 & 1 & 0 & 0 \\
0 & 0 & 0 & 0 & 1 & 0 \\
0 & 0 & 0 & 0 & 0 & 1 \\
0 & 0 & 0 & 0 & 0 & 1 \\
0 & 0 & 0 & 0 & 0 & 0
\end{array}\right), \bar{C}_{2}=\left(\begin{array}{llllll}
0 & 0 & 0 & 0 & 0 & 1 \\
0 & 0 & 0 & 0 & 0 & 1 \\
0 & 1 & 0 & 0 & 0 & 0 \\
1 & 0 & 1 & 0 & 0 & 0 \\
0 & 0 & 1 & 1 & 0 & 0 \\
0 & 0 & 0 & 0 & 0 & 0
\end{array}\right)
\end{aligned}
$$

The proposed method can explore a much wider solution space than the existing GA-based method because the proposed dual chromosome encoding can represent all the possible structures of the BNs. The subsequent theorems show that there is no BN structure that cannot be encoded by the proposed dual genetic encoding and the proposed encoding explores the entire solution space of the BN structures. First, we define two terminologies that will be used in the theorems. The root indicates a node that has no parent nodes and a leaf indicates a node that has no child nodes.

\section{Theorem 1:}

Any DAG has at least one root and one leaf. There is no DAG which does not have a root or a leaf.

Proof. Consider a DAG with $n$ nodes. If there is no leaf in the DAG, then all nodes in the DAG have at least one child node. So if we select any node in the DAG and traverse from the node to one of its child nodes $n+1$ times, then we would encounter the same node at least one time. That means that there is at least one loop in the DAG and this is a contradiction. Therefore, any DAG has at least one leaf. Similarly, if there is no root in the DAG, then all nodes have at least one parent node. If any node in the DAG is selected and movement is in the reverse direction from the node to one of its parent nodes $n+1$ times, then the same node will be encountered at least one time. That means that there is at least one loop in the DAG and this is a contradiction. Therefore, any DAG has at least one root.

\section{Theorem 2:}

Consider a DAG with $n+1$ nodes. The DAG can be decomposed as a connection of a root (or a leaf) and a small DAG with $n$ nodes.

Proof. Consider the DAG $G$ with $n+1$ nodes. Then, by theorem $1, G$ has at least one root and one leaf. If we remove the root $R$ and the connected arcs from $G$, then the resulting graph $G^{\prime}$ will have $n$ nodes. Assume that the resulting graph
$G^{\prime}$ is not the DAG. Then, there is at least one loop in $G^{\prime}$. Even if the deleted node $R$ is appended to $G^{\prime}$ and the disconnected arcs are restored, the resulting graph still has a loop. Since the resulting graph is the original graph $G$ and it has a loop, it is a contradiction. Therefore, $G^{\prime}$ is also the DAG and we can conclude that a DAG with $n+1$ nodes can be decomposed as a connection of a root and a small DAG with $n$ nodes. In the same way, it can be shown that if a leaf $L$ and the connected arcs are removed from $G$, the resulting graph $G^{\prime}$ becomes the DAG and it can be concluded that a DAG with $n+1$ nodes can be decomposed as a combination of a leaf $L$ and a small DAG with $n$ nodes.

Hence, a discussion follows that reveals that there is no BN structure which cannot be encoded by the proposed dual genetic encoding.

\section{Theorem 3:}

Any DAG with $n$ nodes can be represented by a certain ordering among $n$ nodes and an upper triangular connectivity matrix with respect to the given ordering.

Proof. Here, mathematical induction is utilized. When the number of nodes is two, the theorem trivially holds. Assume that $x_{1}$ is a root and $x_{2}$ is a leaf. If the ordering is "12", the connectivity matrix $C=\left(\begin{array}{ll}0 & 1 \\ 0 & 0\end{array}\right)$ for the DAG is upper triangular. Next, assume that this theorem holds when the number of nodes is $n$ and consider a DAG $G$ with $n+1$ nodes. By theorem 2, DAG $G$ with $n+1$ nodes can be decomposed as a connection of a root (or a leaf) and a small DAG with nodes. If $G$ is decomposed as a connection of a root and a small DAG $G^{\prime}$, the root and the connected arcs can be removed. Then, by the assumption, we can find a proper ordering $O$ among $n$ variables and upper triangular connectivity matrix $C$ with respect to the ordering $O$.

$$
\begin{aligned}
O & =a_{1} a_{2} \cdots a_{n} \\
C & =\left(\begin{array}{cccccc}
0 & c_{1,2} & c_{1,3} & \cdots & c_{1, n-1} & c_{1, n} \\
0 & 0 & c_{2,3} & \cdots & c_{2, n-1} & c_{2, n} \\
\vdots & \vdots & \vdots & \ddots & \vdots & \vdots \\
0 & 0 & 0 & \cdots & 0 & c_{n-1, n} \\
0 & 0 & 0 & \cdots & 0 & 0
\end{array}\right)
\end{aligned}
$$

where

$$
\begin{aligned}
& a_{x} \text { is an integer and } a_{x} \in\{1, \cdots, n+1\} \\
& \text { for } x=1, \cdots, n+1 \\
& a_{j} \neq a_{k} \text { iff } j \neq k \\
& c_{i, j}= \begin{cases}1 & \text { if } a_{i} \text { is a parent of } a_{j} \\
0 & \text { otherwise }\end{cases}
\end{aligned}
$$

which fully represents $G^{\prime}$. If the deleted root is denoted as $a_{n+1}$ and the ordering among the $n+1$ variables are selected as

$$
O^{\prime}=a_{n+1} a_{1} a_{2} \cdots a_{n}
$$

then the original DAG with $n+1$ nodes can be represented by an upper triangular connectivity matrix, 


$$
\begin{aligned}
C^{\prime} & =\left(\begin{array}{ccccccc}
0 & c_{n+1,1} & c_{n+1,2} & c_{n+1,3} & \cdots & c_{n+1, n-1} & c_{n+1, n} \\
0 & 0 & c_{1,2} & c_{1,3} & \cdots & c_{1, n-1} & c_{1, n} \\
0 & 0 & 0 & c_{2,3} & \cdots & c_{2, n-1} & c_{2, n} \\
\vdots & \vdots & \vdots & \vdots & \ddots & \vdots & \vdots \\
0 & 0 & 0 & 0 & \cdots & 0 & c_{n-1, n} \\
0 & 0 & 0 & 0 & \cdots & 0 & 0
\end{array}\right) \\
& =\left(\begin{array}{cc}
0 & C_{12}^{\prime} \\
0 & C
\end{array}\right)
\end{aligned}
$$

with respect to the ordering $O^{\prime}=a_{n+1} a_{1} a_{2} \cdots a_{n}$ where

$$
c_{i, j}= \begin{cases}1 & \text { if } a_{i} \text { is a parent of } a_{j} \\ 0 & \text { otherwise }\end{cases}
$$

and $C$ is $n \times n$ upper triangular matrix

and $C_{12}^{\prime}$ is $1 \times n$ matrix

Similarly, if $G$ is decomposed as a connection of a leaf and a small DAG $G^{\prime \prime}$, the leaf and the connected arcs can be removed. Then, by the assumption, a proper ordering $O$ among $n$ variables and upper triangular connectivity matrix $C$ of (11) can be found, which fully represents the small DAG $G^{\prime \prime}$. If the deleted leaf is denoted as $a_{n+1}$ and the ordering among the $n+1$ variables are selected as

$$
O^{\prime \prime}=a_{1} a_{2} \cdots a_{n} a_{n+1}
$$

then the original DAG with $n+1$ nodes can be represented by an upper triangular connectivity matrix,

$$
\begin{aligned}
C^{\prime \prime} & =\left(\begin{array}{ccccccc}
0 & c_{1,2} & c_{1,3} & c_{1,4} & \cdots & c_{1, n} & c_{1, n+1} \\
0 & 0 & c_{2,3} & c_{2,4} & \cdots & c_{2, n} & c_{2, n+1} \\
0 & 0 & 0 & c_{3,4} & \cdots & c_{3, n} & c_{3, n+1} \\
\vdots & \vdots & \vdots & \vdots & \ddots & \vdots & \vdots \\
0 & 0 & 0 & 0 & \cdots & 0 & c_{n, n+1} \\
0 & 0 & 0 & 0 & \cdots & 0 & 0
\end{array}\right) \\
& =\left(\begin{array}{cc}
C & C_{12}^{\prime \prime} \\
0 & 0
\end{array}\right)
\end{aligned}
$$

where

$$
\begin{aligned}
& c_{i, j}= \begin{cases}1 & \text { if } a_{i} \text { is a parent of } a_{j} \\
0 & \text { otherwise }\end{cases} \\
& \text { and } C \text { is } n \times n \text { upper triangular matrix } \\
& \text { and } C_{12}^{\prime \prime} \text { is } n \times 1 \text { matrix }
\end{aligned}
$$

This means that if the property is true for $n$, the property is also true for $n+1$ and the proof is completed.

\subsubsection{Crossover}

Consider the genetic operations (crossover and mutation) for the dual chromosome. First, let us consider the crossover. In the case of connectivity matrix, any upper triangular matrix is valid as a BN structure. Thus, the plain crossover is enough and is closed in the domain of the connectivity chromosomes as in [7]. However, in the case of the ordering chromosome, the crossover is not straightforward. In order to explain the genetic crossover for the ordering chromosomes, let $X_{o}$ and $Y_{o}$ be two $n$ dimensional ordering chromosomes represented by

$$
\begin{aligned}
& X_{o}=x_{1} x_{2} \cdots x_{n} \\
& Y_{o}=y_{1} y_{2} \cdots y_{n}
\end{aligned}
$$

The proposed crossover generates a random number $r$ from a uniform distribution from 1 to $n$ and creates two new chromosomes $X_{o}^{\prime}$ and $Y_{o}^{\prime}$ according to following equations.

$$
\begin{aligned}
& X_{o}^{\prime}=x_{1}^{\prime} x_{2}^{\prime} \cdots x_{n}^{\prime} \\
& Y_{o}^{\prime}=y_{1}^{\prime} y_{2}^{\prime} \cdots y_{n}^{\prime} \\
& x_{i}^{\prime}= \begin{cases}x_{i} & \text { if } i<r \\
y_{\alpha_{i-r}} & \text { otherwise }\end{cases} \\
& y_{i}^{\prime}= \begin{cases}y_{i} & \text { if } i<r \\
x_{\beta_{i-r}} & \text { otherwise }\end{cases}
\end{aligned}
$$

where

$$
\begin{aligned}
& \alpha_{0}, \alpha_{1}, \cdots, \alpha_{n-r} \in\{1,2, \cdots, n\} \\
& \alpha_{0}<\alpha_{1}<\cdots<\alpha_{n-r} \\
& y_{\alpha_{j}} \neq x_{1}, x_{2}, \cdots, x_{r-1} \text { for } j=0,1,2, \cdots, n-r \\
& \beta_{0}, \beta_{1}, \cdots, \beta_{n-r} \in\{1,2, \cdots, n\} \\
& \beta_{0}<\beta_{1}<\cdots<\beta_{n-r} \\
& x_{\beta_{j}} \neq y_{1}, y_{2}, \cdots, y_{r-1} \text { for } j=0,1,2, \cdots, n-r
\end{aligned}
$$

Example 2 (Crossover for ordering chromosome):

Consider the two BNs in Example 1. Assume that the crossover operation is applied to the ordering chromosomes at the point between the second and the third genes, which means $r=3$ and $n=6$ in this example. The offspring start with

$$
\begin{aligned}
& X_{1 o}^{\prime}=12 \mathrm{xxxx} \\
& X_{2 o}^{\prime}=54 \mathrm{xxxx}
\end{aligned}
$$

Since $X_{1 o}^{\prime}$ contains " 1 " and " 2 ", the two digits in $X_{2 o}$ are deleted. Then the remaining sub-chromosome of $X_{2 o}$ is "5436." The concatenation of the two parts yields an offspring ordering chromosome $X_{1 o}^{\prime}=125436$. In the same way, " 54 " and " 1263 " are concatenated and the another offspring ordering chromosome becomes $X_{2 o}^{\prime}=541263$.

$$
\begin{aligned}
& X_{1 o}^{\prime}=125436 \\
& X_{1 c}^{\prime}=110000100010010 \\
& X_{2 o}^{\prime}=541263 \\
& X_{2 c}^{\prime}=110001100010011
\end{aligned}
$$

$$
C_{1}^{\prime}=\left(\begin{array}{llllll}
0 & 1 & 1 & 0 & 0 & 0 \\
0 & 0 & 0 & 1 & 0 & 0 \\
0 & 0 & 0 & 0 & 1 & 0 \\
0 & 0 & 0 & 0 & 0 & 1 \\
0 & 0 & 0 & 0 & 0 & 0 \\
0 & 0 & 0 & 0 & 0 & 0
\end{array}\right), C_{2}^{\prime}=\left(\begin{array}{llllll}
0 & 1 & 1 & 0 & 0 & 0 \\
0 & 0 & 1 & 1 & 0 & 0 \\
0 & 0 & 0 & 0 & 1 & 0 \\
0 & 0 & 0 & 0 & 0 & 1 \\
0 & 0 & 0 & 0 & 0 & 1 \\
0 & 0 & 0 & 0 & 0 & 0
\end{array}\right)
$$

If the connectivity chromosomes are the same as the parents, then the offspring BNs are depicted as in Fig. 3. 

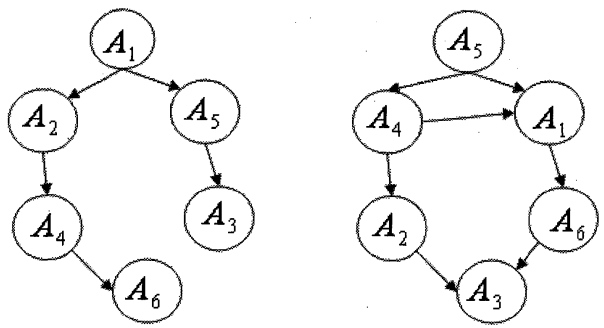

Fig. 3 Offspring BNs after crossover (Example 2).

The following shows the pseudo code of crossover for the ordering chromosome:

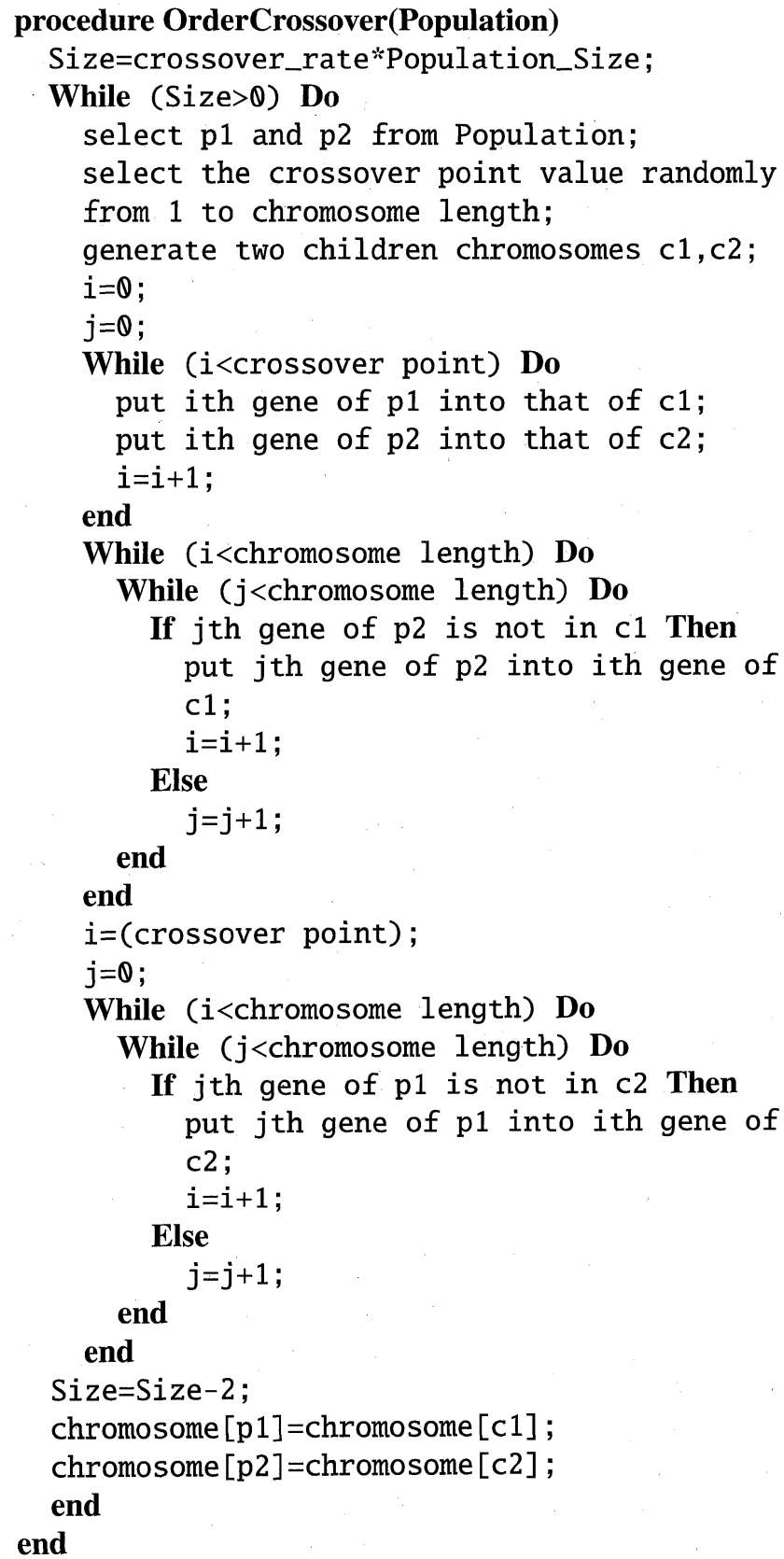

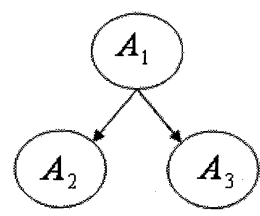

(a)

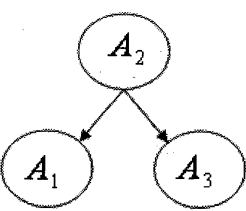

(b)
Fig. 4 Two BNs before and after mutation (Example 3).

\subsubsection{Mutation}

For the connectivity chromosome, the plain mutation produces only the upper triangular matrix as in case of the crossover operation. Thus, the plain mutation is enough and is closed in the domain of the connectivity chromosomes as in [7]. The ordering chromosome is not the case. The following discussion explains the genetic mutation for the ordering chromosome. Let $X_{o}$ be the $n$ dimensional ordering chromosome,

$$
X_{o}=x_{1} x_{2} \cdots x_{n}
$$

The proposed mutation operator generates a random number $r_{1}$ and $r_{2}$ from a uniform distribution from 1 to $n$ and creates the new chromosomes $X_{o}^{\prime}$ according to following equations:

$$
\begin{aligned}
& X_{o}^{\prime}=x_{1}^{\prime} x_{2}^{\prime} \cdots x_{n}^{\prime} \\
& x_{i}^{\prime}= \begin{cases}x_{r_{2}} & \text { if } i=r_{1} \\
x_{r_{1}} & \text { else if } i=r_{2} \\
x_{i} & \text { otherwise }\end{cases}
\end{aligned}
$$

Example 3 (Mutation for ordering chromosome):

Consider a BN structure with three variables as shown in Fig. 4 (a).

Using the proposed dual genetic encoding, the ordering and the connectivity chromosomes are represented as:

$$
\begin{aligned}
& X_{o}=123 \\
& X_{c}=110
\end{aligned}
$$

respectively, and the connectivity matrix is as follows.

$$
C=\left(\begin{array}{lll}
0 & 1 & 1 \\
0 & 0 & 0 \\
0 & 0 & 0
\end{array}\right)
$$

Suppose that the first and the second genes of the ordering chromosome are exchanged, which means $r_{1}=1, r_{2}=2$, and $n=3$ in this example, and the connectivity chromosome does not change. The resulting dual chromosome and the connectivity matrix become:

$$
\begin{aligned}
& X_{o}^{\prime}=213 \\
& X_{c}^{\prime}=110 \\
& C^{\prime}=\left(\begin{array}{lll}
0 & 1 & 1 \\
0 & 0 & 0 \\
0 & 0 & 0
\end{array}\right)
\end{aligned}
$$

Fig. 4 (b) depicts the mutated BN. 
The following shows the pseudo code of mutation operation for the ordering chromosome:

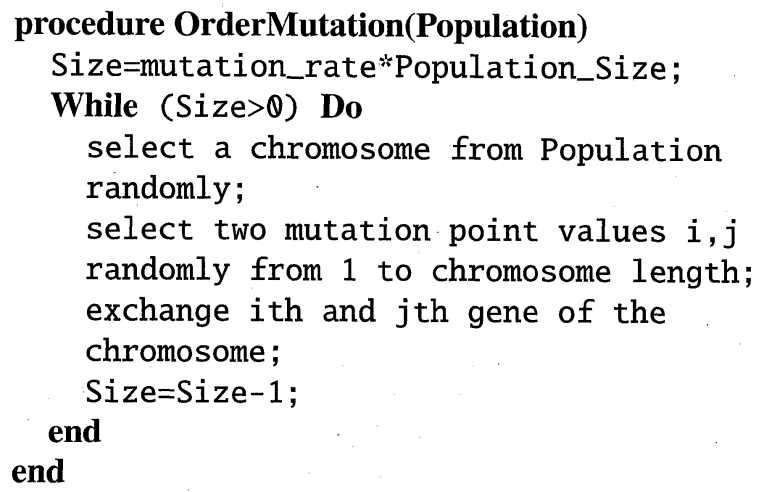

\section{Simulation}

In this section, the proposed method is applied to three realworld problems and the validity of this method is demonstrated through computer simulation. In all the simulations, we restrict the maximum number of parents of each node to five. The restriction is not necessary but we place it for simple implementation. Without the restrictions, the number of possible configurations would increase exponentially with the number of parents [21]. It is a trade off between the performance and the computational cost.

\subsection{Home Network System}

In this section, the proposed method is applied to the home network system. The home network system is a typical test bed of the ubiquitous computing and sensor network, while it is expected to upgrade the quality of living over approximately the next decade. The implementation of contextaware computing is the key issue in the home network and has received attention from engineers and scientists. Context-aware computing is jargon coined by computer scientists and it is aimed at

1. gathering information about the users from various sensors distributed in a smart home,

2. recognizing the intention of the users, and

3. providing them with human friendly services.

In context-aware computing, it is assumed that

1. the home appliances and devices are equipped with wired/wireless sensors,

2. they gather information about the circumstances under which they operate,

3. they presume the intention or action of the users, and

4. they react according to the intention of the users.

Literature provides that the contexts of user activities can be presumed from the state of the environment [19]. However, context-awareness is not fully understood and the tools and techniques for developing context-aware applications

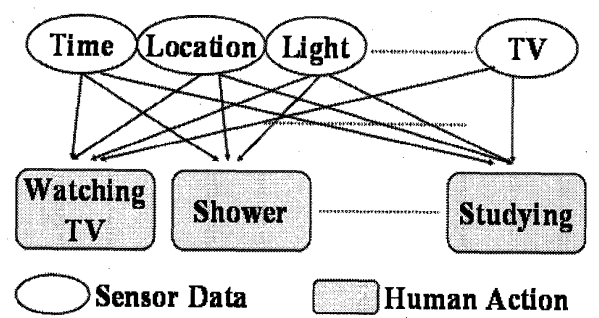

Fig. 5 An example of the BN structure for a Home network system.

are still in their infancy. BNs are one of the possible tools for the context-aware computing. In this example, the proposed method is applied and a BN is developed that presumes the intention or action of a single user, who lives in the home network system. Figure 5 shows the example of BN structure of the context-aware system for the home network.

A web-based virtual action simulator was developed in FLASH and a database of 672 cases for a context-aware system was built, for the purpose of training the $\mathrm{BN}$ [13]. Forty two random variables are used to implement the contextawareness of the BN. Twenty one variables represent the state of the home appliances such as the TV, lights in the living room, the refrigerator, etc. The other twenty one variables represent the activities of a single user, for example whether he/she is studying, sleeping, or dressing. Using the proposed method, the Bayesian network is trained such that the action of the user is presumed from the measurements of the sensors distributed in the context-aware home.

\subsection{Car Diagnosis Problems}

The car diagnosis problem introduced by Norsys is a simple example of belief network. The reason why a car does not move is presumed, based on spark plugs, headlights, main fuse, etc [14]. Eighteen nodes are used in this BN and all nodes of the network take discrete values. Some of them can take on three discrete states and the others can take on two states. A database of two thousand cases is utilized to train the BN. The database was generated from Netica tool [14]. Figure 6 shows the structure of the car diagnosis problem depicted by Netica from which the sample cases were collected. Note that, Fig. 6 depicts the desired BN structure and the goal of the structure learning for $\mathrm{BN}$ is to obtain the structures which are close to this desired one.

\subsection{ALARM Network}

ALARM (A Logical Alarm Reduction Mechanism) is a medical diagnostic system for patient monitoring. It is a complex belief network with eight diagnoses, sixteen findings, and thirteen intermediate variables [20]. A database of two thousand cases is utilized to train the BN. As in the previous example, the database is generated from Netica tool [14]. Figure 7 shows the structure of the ALARM network depicted by Netica from which the sample cases are collected. Note that, Fig. 10 depicts the desired BN structure 


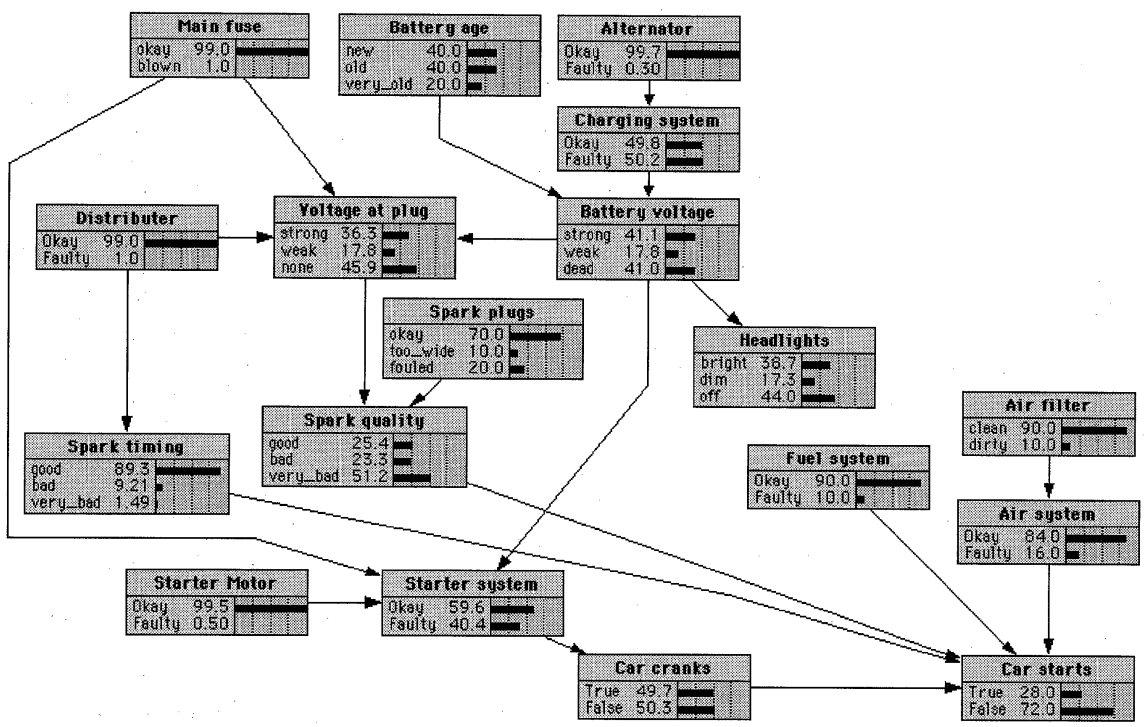

Fig. 6 The structure of the Car diagnosis problem network.

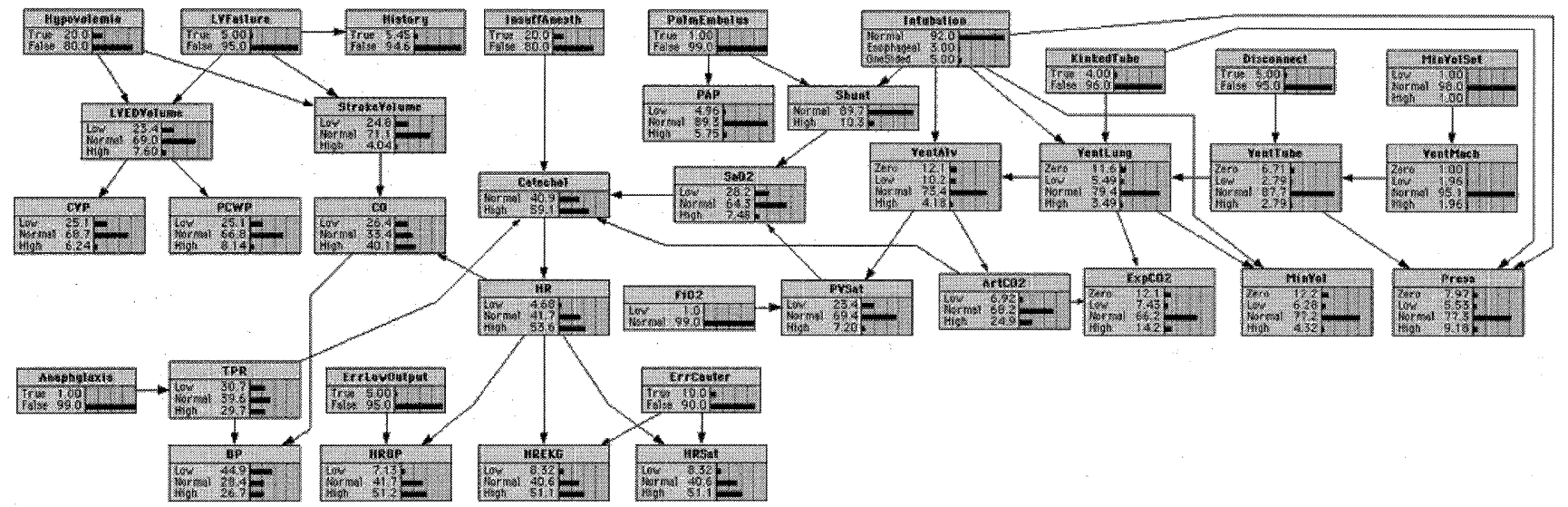

Fig. 7 The structure of the ALARM network.

and the goal of the structure learning for $\mathrm{BN}$ is to obtain the structures which are close to this desired one.

\subsection{Simulation Results}

Using the three databases, the BNs were built to represent the probabilistic relationships between the variables. Theorem 4 was utilized to apply the proposed dual genetic algorithms and evaluate the validity of the structure of the given $\mathrm{BN}[10]$.

\section{Theorem 4:}

Let $Z$ be a set of $n$ discrete variables, where a variable $A_{i}$, in $Z$ has $r_{i}$ possible value assignments: $\left(v_{i 1}, \cdots, v_{i r_{i}}\right)$. Let $D$ be a database of $m$ cases, where each case contains a value assignment for each variable in $Z$. Let $B_{s}$ denote a belief network structure containing just the variables in $Z$. Each variable $A_{i}$, in $B_{s}$ has a set of parents, which are represented with a list of variables $p a\left(A_{i}\right)$. Let $w_{i} j$ denote the $j$ th unique instantiation of $p a\left(A_{i}\right)$ relative to $D$. Suppose there are $q_{i}$ such unique instantiations of $p a\left(A_{i}\right)$. Define $N_{i} j k$ to be the number of cases in $D$ in which variable $A_{i}$ has the value $v_{i k}$ and $p a\left(A_{i}\right)$ is instantiated as $w_{i} j$. Let $N_{i j}=\sum_{k=1}^{r_{i}} N_{i j k}$. If the cases occur independently and the probability density function $f\left(B_{p} \mid B_{s}\right)$ is uniform, where $B_{p}$ is a vector whose values denote the conditional probability assignments associated with belief network structure $B_{s}$, then it follows that

$$
P\left(B_{s}, D\right)=P\left(B_{s}\right) \prod_{i=1}^{n} \prod_{j=1}^{q_{i}} \frac{\left(r_{i}-1\right) !}{\left(N_{i j}+r_{i}-1\right) !} \prod_{k=1}^{r_{i}} N_{i j k} !
$$

Since $P\left(B_{s}, D\right)=P\left(B_{s}\right) P\left(D \mid B_{s}\right)$ and $P\left(B_{s}\right)$ is uniformly distributed, the fittest $\mathrm{BN}$ structure $B_{s}$ is determined by maximizing

$$
P\left(D \mid B_{s}\right)=\prod_{i=1}^{n} \prod_{j=1}^{q_{i}} \frac{\left(r_{i}-1\right) !}{\left(N_{i j}+r_{i}-1\right) !} \prod_{k=1}^{r_{i}} N_{i j k} !
$$

The simulation was run ten times for each problem and the parameters of the simulations were as follows: the population size of GA was 50 , the crossover rate was 0.65 and 


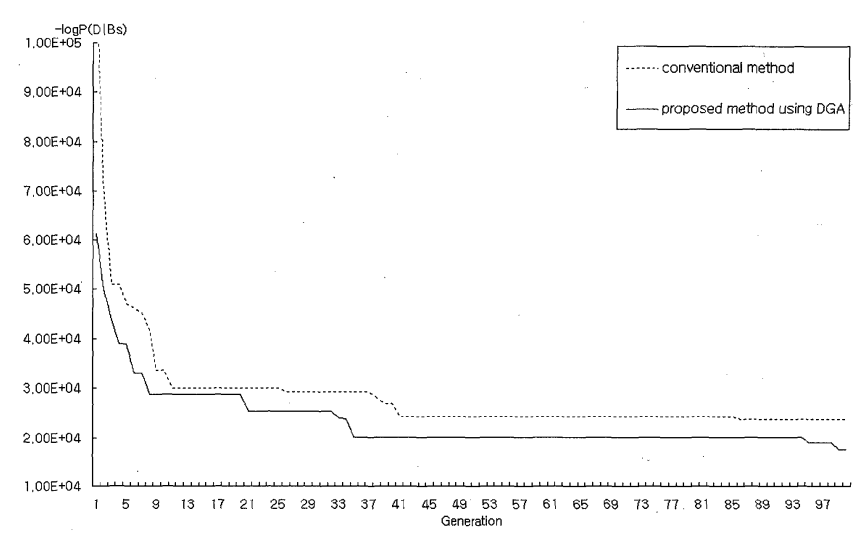

Fig. 8 Performance of the conventional and proposed methods (Home network system).

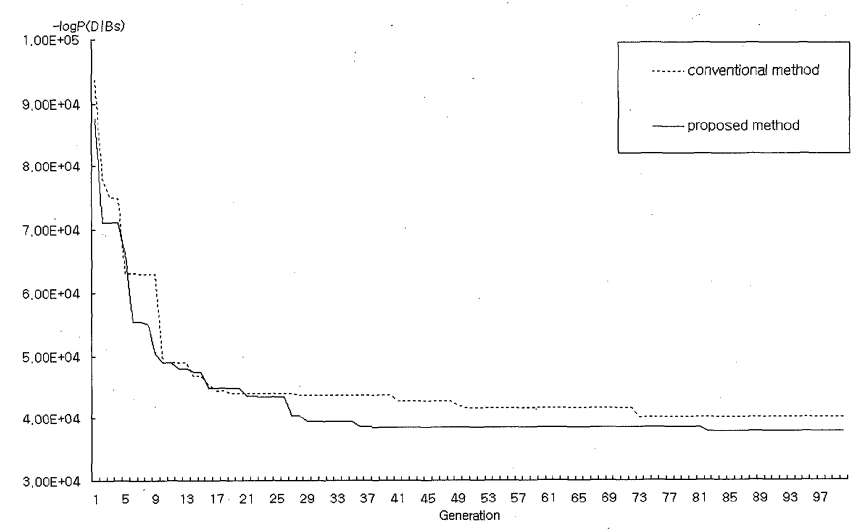

Fig. 9 Performance of the conventional and proposed methods (Car diagnosis problem).

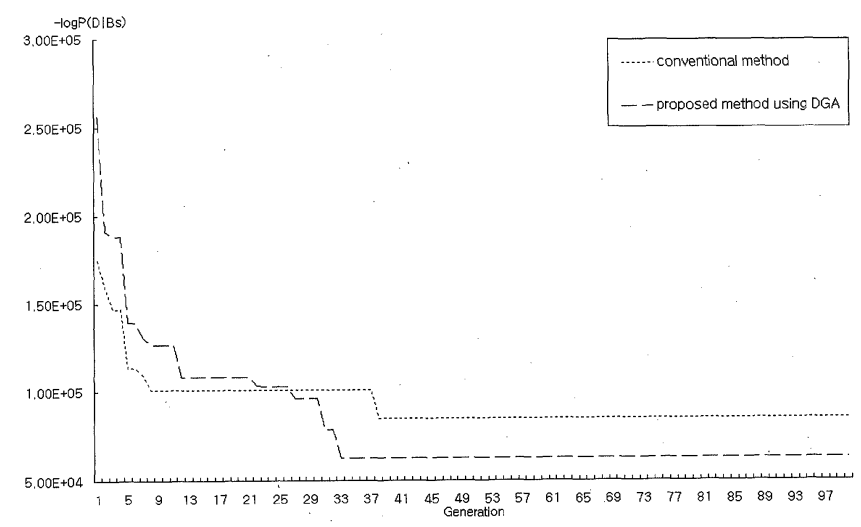

Fig. 10 Performance of the conventional and proposed methods (ALARM network).

the mutation rate was 0.05 . The formula in Theorem 4 was used, as an objective function to be maximized. The algorithms were stopped when 5,000 structures had been evaluated, which means that 100 generations have been evaluated. In this simulation, we assume that there is no prior knowledge about the node ordering or the casual relationship between the nodes. Therefore, we randomly select the order of the nodes in the conventional method. Figures 8,9 , and
Table 1 Comparison of the proposed and the previous methods (Home network problem).

\begin{tabular}{|c|c|l|c|}
\hline Application & Trial time & $\begin{array}{l}\text { Conventional } \\
\text { method }\end{array}$ & Proposed method \\
\hline \multirow{5}{*}{} & 1 & 23895.26 & 17823.24 \\
\cline { 2 - 4 } & 2 & 27617.36 & 20605.52 \\
\cline { 2 - 4 } & 3 & 20809.5 & 19482.32 \\
\cline { 2 - 4 } Home & 4 & 19119.23 & 20047.76 \\
\cline { 2 - 4 } network & 5 & 20850.03 & 21245.77 \\
\cline { 2 - 4 } & 6 & 21808.97 & 19360.47 \\
\cline { 2 - 4 } & 7 & 18104.05 & 21817.03 \\
\cline { 2 - 4 } & 8 & 21760.08 & 22136.7 \\
\cline { 2 - 4 } & 9 & 24102.79 & 21767.96 \\
\cline { 2 - 4 } & 10 & 24806.22 & 20328.63 \\
\cline { 2 - 4 } & Average & 22287.35 & 20461.54 \\
\cline { 2 - 4 } & Variance & 8103187 & 1811859 \\
\cline { 2 - 4 } & Best & 18104.05 & 17823.24 \\
\cline { 2 - 4 } & Worst & 27617.36 & 22136.7 \\
\hline
\end{tabular}

Table 2 Comparison of the proposed and the previous methods (Car diagnosis problem).

\begin{tabular}{|c|c|l|c|}
\hline Application & Trial time & $\begin{array}{l}\text { Conventional } \\
\text { method }\end{array}$ & Proposed method \\
\hline \multirow{5}{*}{} & 1 & 39687.91 & 37821.28 \\
\cline { 2 - 4 } & 2 & 41628.53 & 38468.16 \\
\cline { 2 - 4 } & 3 & 43565.91 & 39754.24 \\
\cline { 2 - 4 } diagnosis & 4 & 42368.24 & 37821.28 \\
\cline { 2 - 4 } problem & 5 & 38214.65 & 37821.28 \\
\cline { 2 - 4 } & 6 & 39658.21 & 37923.45 \\
\cline { 2 - 4 } & 7 & 39462.29 & 38165.23 \\
\cline { 2 - 4 } & 8 & 40216.35 & 37821.28 \\
\cline { 2 - 4 } & 9 & 39845.23 & 37821.28 \\
\cline { 2 - 4 } & Average & 41268.24 & 38321.46 \\
\cline { 2 - 4 } & Variance & 2538557.56 & 38173.89 \\
\cline { 2 - 4 } & Best & 38214.65 & 365593.57 \\
\cline { 2 - 4 } & Worst & 43565.91 & 37821.28 \\
\hline
\end{tabular}

10 show the learning result of the first run for the home network, car diagnosis, and ALARM problems, respectively. In the figures, the dashed lines represent the result of the conventional method and the solid lines represent the result of the proposed method. The vertical axis is the negative logarithm of (34) and the horizontal axis is the number of generations. As BN attempts to approximate the entire database, the vertical axis approaches zero, since the probability of one corresponds to zero on the negative logarithm axis. In the home network and car diagnostic systems, it can be seen that the proposed method initially outperforms the conventional method and continues to outperform the conventional method for the next one hundred generations, as shown in Figs. 8 and 9, respectively.

Although the proposed method does not show good performance for less than thirty generations, it outperforms the conventional method after thirty generations in the ALARM network, as shown in Fig. 10.

The reason for the excellence of the proposed method could be attributed to the fact that the search space was fully explored by the dual encoding of GA. Tables 1, 2, and 3 compare the performances of the conventional method and the proposed method in terms of (34). The simulation was 
Table 3 Comparison of the proposed and the previous methods (Home network problem).

\begin{tabular}{|c|c|c|c|}
\hline Application & Trial time & $\begin{array}{l}\text { Conventional } \\
\text { method }\end{array}$ & Proposed method \\
\hline \multirow{14}{*}{$\begin{array}{l}\text { ALARM } \\
\text { network }\end{array}$} & 1 & 86548.32 & 73401.54 \\
\hline & 2 & 84231.24 & 75860.24 \\
\hline & 3 & 79324.23 & 73401.54 \\
\hline & 4 & 86954.94 & 73401.54 \\
\hline & 5 & 84321.12 & 78203.38 \\
\hline & 6 & 81423.24 & 73401.54 \\
\hline & 7 & 75165.21 & 73401.54 \\
\hline & 8 & 84246.73 & 74632.21 \\
\hline & 9 & 81234.23 & 75352.21 \\
\hline & 10 & 87549.65 & 73401.54 \\
\hline & Average & 83099.891 & $74445: 73$ \\
\hline & Variance & 14905182.13 & 2613249.931 \\
\hline & Best & 75165.21 & 73401.54 \\
\hline & Worst & 87549.65 & 78203.38 \\
\hline
\end{tabular}

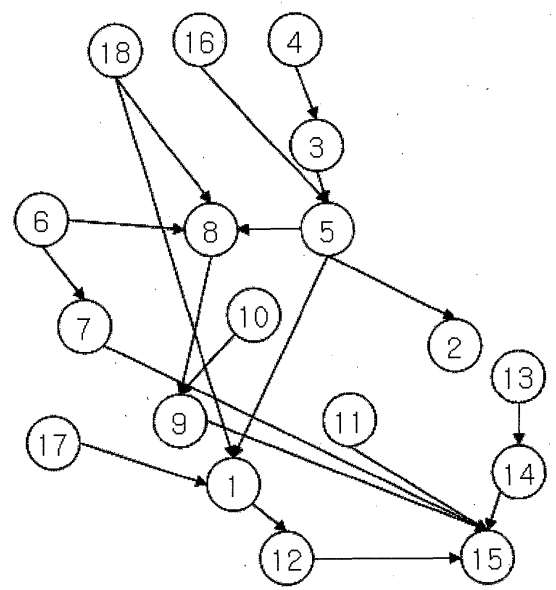

Fig. 11 Network structure of the proposed learning result (Car diagnosis problem).

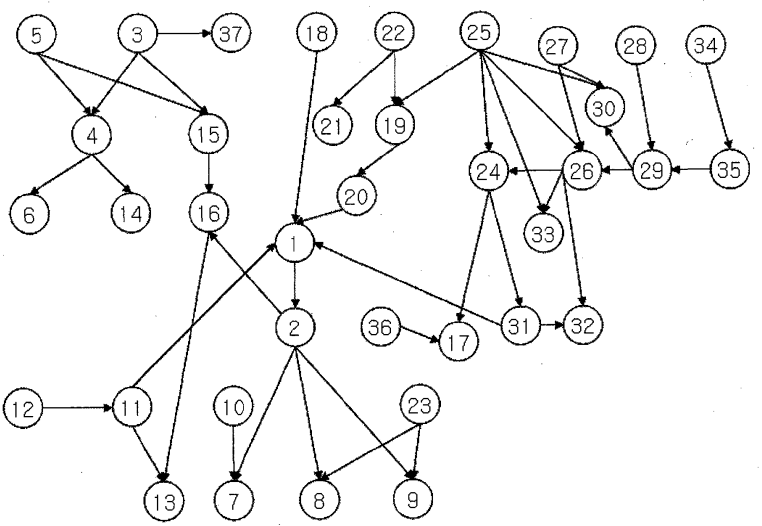

Fig. 12 structure of the proposed learning result (ALARM network).

run until 5,000 structures had been evaluated. This was repeated ten times. For all three problems, the proposed method outperforms the conventional method in not only the average case but also in the best and worst cases.

Figures. 11 and 12 show the network structures for the learning results of the first run in case of Car diagnosis prob-
Table 4 Index and name of the nodes (Car diagnosis problem).

\begin{tabular}{|c|c|c|c|}
\hline Node index & Node name & Node index & Node name \\
\hline 1 & Starter system & 10 & Spark plugs \\
\hline 2 & Headlights & 11 & Fuel system \\
\hline 3 & Charging system & 12 & Car cranks \\
\hline 4 & Alternator & 13 & Air filter \\
\hline 5 & Battery voltage & 14 & Air system \\
\hline 6 & Distributer & 15 & Car starts \\
\hline 7 & Spark timing & 16 & Battery age \\
\hline 8 & Voltage at plug & 17 & Starter motor \\
\hline 9 & Spark quality & 18 & Main fuse \\
\hline
\end{tabular}

Table 5 Index and name of the nodes (ALARM network).

\begin{tabular}{|c|c|c|c|}
\hline Node index & Node name & Node index & Node name \\
\hline 1 & Catechol & 20 & SaO2 \\
\hline 2 & HR & 21 & PAP \\
\hline 3 & LVFailure & 22 & PulmEmbolus \\
\hline 4 & LVEDVolume & 23 & ErrCauter \\
\hline 5 & Hypovolemia & 24 & VentAlv \\
\hline 6 & CVP & 25 & Intubation \\
\hline 7 & HRBP & 26 & VentLung \\
\hline 8 & HREKG & 27 & KinkedTube \\
\hline 9 & HRSat & 28 & Disconnect \\
\hline 10 & ErrLowOutput & 29 & VentTube \\
\hline 11 & TPR & 30 & Press \\
\hline 12 & Anaphylaxis & 31 & ArtCO2 \\
\hline 13 & BP & 32 & ExpCO2 \\
\hline 14 & PCWP & 33 & MinVol \\
\hline 15 & StrokeVolume & 34 & MinVolSet \\
\hline 16 & CO & 35 & VentMach \\
\hline 17 & PVSat & 36 & FiO2 \\
\hline 18 & InsuffAnesth & 37 & History \\
\hline 19 & Shunt & & \\
\hline \multicolumn{4}{|r}{} \\
\hline
\end{tabular}

Table 6 Statistical results of the conventional and proposed method.

\begin{tabular}{|l|c|l|l|l|}
\hline Application & Algorithm & Best & Mean & Variance \\
\hline \multirow{2}{*}{$\begin{array}{l}\text { Car } \\
\text { diagnosis } \\
\text { problem }\end{array}$} & Conventional & 38214.65 & 40591.56 & 2538557.87 \\
\cline { 2 - 5 } & Proposed & 37821.28 & 38173.89 & 365593.57 \\
\cline { 2 - 5 } & Target & \multicolumn{3}{|c|}{37821.28} \\
\hline \multirow{3}{*}{$\begin{array}{l}\text { ALARM } \\
\text { network }\end{array}$} & Conventional & 75165.21 & 83099.89 & 14905182.13 \\
\cline { 2 - 5 } & Proposed & 73401.54 & 74445.73 & 2613249.93 \\
\cline { 2 - 5 } $\begin{array}{l}\text { Home } \\
\text { network } \\
\text { system }\end{array}$ & Target & \multicolumn{4}{|c|}{73401.54} \\
\cline { 2 - 5 } & Conventional & 18104.05 & 22287.35 & 8103187 \\
\cline { 2 - 5 } & Proposed & 17823.24 & 20461.54 & 1811859 \\
\hline
\end{tabular}

lem and ALARM network, respectively. It can be shown that the resulting structures are same as the target network structures in Figs. 6 and 7, respectively. Tables 4 and 5 represent the indices and names of the node which are shown in Figs. 11 and 12, respectively.

Statistical results are summarized in Table 6. In the table, the learned structures are compared with the target structure from which the sample data are taken. In case of the Home network system, there is no target structure since the data are collected not from the target structure but from the virtual simulator.

For further evaluation, we compare the proposed method with the conventional method in terms of number of arcs correctly added between the same nodes as those in the target network, the number of missed arcs of the target network, or the number of extra arcs added wrongly. In Tables 7 
Table 7 Comparison with the target structure (Car diagnosis problem).

\begin{tabular}{|l|l|l|l|l|l|}
\hline \multirow{4}{*}{ Application } & Trial & \multicolumn{2}{|l|}{ Conventional method } & \multicolumn{2}{c|}{ Proposed method } \\
\cline { 3 - 6 } & & $\begin{array}{l}\text { missed } \\
\text { arcs }\end{array}$ & $\begin{array}{l}\text { wrongly } \\
\text { added arcs }\end{array}$ & $\begin{array}{l}\text { missed } \\
\text { arcs }\end{array}$ & $\begin{array}{l}\text { wrongly } \\
\text { added arcs }\end{array}$ \\
\hline \multirow{5}{*}{$\begin{array}{l}\text { Car } \\
\text { diagnosis } \\
\text { problem }\end{array}$} & 1 & 2 & 2 & 0 & 0 \\
\cline { 2 - 6 } & 2 & 4 & 2 & 1 & 2 \\
\cline { 2 - 6 } & 3 & 5 & 1 & 2 & 1 \\
\cline { 2 - 6 } & 4 & 3 & 3 & 0 & 0 \\
\cline { 2 - 6 } & 5 & 1 & 0 & 0 & 0 \\
\cline { 2 - 6 } & 6 & 1 & 1 & 0 & 1 \\
\cline { 2 - 6 } & 7 & 2 & 0 & 1 & 1 \\
\cline { 2 - 6 } & 8 & 3 & 0 & 0 & 0 \\
\cline { 2 - 6 } & 9 & 3 & 0 & 0 & 0 \\
\cline { 2 - 6 } & 10 & 4 & 0 & 1 & 0 \\
\hline
\end{tabular}

Table 8 Comparison with the target structure (ALARM network).

\begin{tabular}{|l|c|l|l|l|l|}
\hline \multirow{2}{*}{ Application } & \multirow{2}{*}{$\begin{array}{l}\text { Trial } \\
\text { time }\end{array}$} & \multicolumn{2}{|c|}{ Conventional method } & \multicolumn{2}{c|}{ Proposed method } \\
\cline { 3 - 6 } & & $\begin{array}{l}\text { missed } \\
\text { arcs }\end{array}$ & $\begin{array}{l}\text { wrongly } \\
\text { added arcs }\end{array}$ & $\begin{array}{l}\text { missed } \\
\text { arcs }\end{array}$ & $\begin{array}{l}\text { wrongly } \\
\text { added arcs }\end{array}$ \\
\hline \multirow{4}{*}{$\begin{array}{l}\text { Car } \\
\text { diagnosis } \\
\text { problem }\end{array}$} & 1 & 5 & 2 & 0 & 0 \\
\cline { 2 - 6 } & 2 & 4 & 1 & 2 & 0 \\
\cline { 2 - 6 } & 3 & 4 & 2 & 0 & 0 \\
\cline { 2 - 6 } & 4 & 6 & 1 & 0 & 0 \\
\cline { 2 - 6 } & 5 & 4 & 0 & 3 & 1 \\
\cline { 2 - 6 } & 6 & 3 & 0 & 0 & 0 \\
\cline { 2 - 6 } & 7 & 3 & 0 & 0 & 0 \\
\cline { 2 - 6 } & 8 & 4 & 1 & 1 & 1 \\
\cline { 2 - 6 } & 9 & 3 & 2 & 1 & 2 \\
\cline { 2 - 6 } & 10 & 7 & 2 & 0 & 0 \\
\hline
\end{tabular}

and 8, the two methods are compared. It can be seen that the proposed method finds the target network much better than the conventional method and the reason might be that the proposed method fully explore the entire search space for the fittest structure.

\section{Conclustions}

In this paper, a new structure learning approach for BNs has been proposed. In this method, the $\mathrm{BN}$ is encoded as a dual chromosome that is composed of an ordering chromosome and a connectivity chromosome. It is rigorously shown that the proposed dual genetic encoding fully represents the possible BN structure. A wider solution space is explored than in previous method. The proposed method is applied to three real-world and benchmark problems, while simulation reveals improved performance over the previous method for all three case studies. In this experimentation, it should be noted that the cost function (34) taken from [10] does not consider the computation complexity and the cases that there are some unobserved nodes. If we can devise more reasonable cost function which overcomes these problems, we can obtain better BN structure. Finally, this is recommended as a future work.

\section{References}

[1] F.V. Jensen, "Introduction to Bayesian networks," Technical Report IR 93-2003, Dept. of Mathematics and Computer Science, Univ. of Aalborg, Denmark, 1993.

[2] M.L. Wong and K.S. Leung, "An efficient data mining method for learning Bayesian networks using an evolutionary algorithm-based hybrid approach," IEEE Trans. Evol. Comput., vol.8, no.4, pp.378404, Aug. 2004.

[3] H. Wang, D. Dash, and M.J. Druzdzel, "A method for evaluating elicitation schemes for probabilistic models," IEEE Trans. Syst. Man Cybern. B, vol.32, no.1, pp.38-43, Feb. 2002.

[4] S. Acid, L.M. De Campos, A. Gonzalez, R. Molina, and N. Perez de la Blanca, "Learning with CASTLE," in Symbolic and Quantitative Approaches to Uncertainty, ed. R. Kruse and P. Siegel, LNCS 548, Springer-Verlag, 1991.

[5] D.M. Chickering, D. Geiger, and D. Heckerman, "Learning Bayesian networks: Search methods and experimental results," Fifth Int'l Workshop Artificial Intelligence and Statistics, pp.112-128, 1995.

[6] G.M. Provan, "Model selection for diagnosis and treatment using temporal influence diagrams," Proc. Fourth Int'1 Workshop Artificial Intelligence and Statistics, pp.469-480, 1995.

[7] P. Larrañaga, M. Poza, Y. Yurramendi, R. Murga, and C. Kuijpers, "Structure learning of Bayesian network by genetic algorithms: A performance analysis of control parameters," IEEE Trans. Pattern Analysis and Machine Intelligence, vol.18, no.9, pp.912-926, Sept. 1996.

[8] R. Garza-Domínguez, M. Martínez-Morales, N. Cruz-Ramírez, J.L. Jiménez-Andrade and A.G. Hernández, "A method based on genetic algorithms and fuzzy logic to induce bayesian networks," Proc. Fifth Mexican International Conference in Computer Science (ENC2004), pp.176-180, Colima, Mexico, 2004.

[9] L.M.d. Campos, J.A. Gámez, and S. Moral, "Partial abductive inference in Bayesian belief networks - An evolutionary computation approach by using problem-specific genetic operators," IEEE Trans. Evol. Comput., vol.6, no.2, pp.105-131, April 2002.

[10] G.F. Cooper and E.A. Herskovits, "A Bayesian method for the induction of probabilistic networks from data," Mach. Learn., vol.9, no.4, pp.309-347, 1992

[11] X. Li, X. He, and S. Yuan, "Learning Bayesian networks structures from incomplete data based on extending evolutionary programming," Proc. 2005 International Conference Machine Learning and Cybernetics 2005, vol.4, pp.2039-2043, Aug. 2005.

[12] S. Shetty and M. Song, "Structure learning of Bayesian networks using a semantic genetic algorithm-based approach," 3rd International Conference Information Technology: Research and Education 2005 (ITRE 2005), pp.454-458, June 2005.

[13] W. Chung, Context-aware Application for Smart Home Based on Bayesian Network, Master Thesis, Yonsei University, 2006.

[14] http://www.norsys.com/networklibrary.html

[15] F.V. Jensen, Bayesian Networks and Decision Graphs, Springer, 2001.

[16] S.Z. Zhang, Z.N. Zhang, N.H. Yang, J.Y. Zhang, and X.K. Wang, "An improved EM algorithm for Bayesian networks parameter learning," Mach. Learn. Cybern., vol.3, pp.1503-1508, Aug. 2004

[17] Z. Michalewicz, Genetic Algorithms + Data Structures = Evolution Programs, Springer, 1999.

[18] P. Larrañaga, C. Kuijpers, and R. Murga, "Learning Bayesian network structures by searching for the best ordering with genetic algorithms," IEEE Trans. Syst. Man Cybern. A, vol.26, no.4, pp.487493, July 1996.

[19] P. Korpipää, M. Koskinen, J. Peltola, S. Mäkelä, and T. Seppänen, "Bayesian approach to sensor-based context awareness," Personal and Ubiquitous Computing J., vol.7, no.4, pp.113-124, July 2003.

[20] I.A. Beinlinch, H.J. Suermondt, R.M. Chavez, and G.F. Cooper, "The ALARM monitoring system: A case study with two probabilistic inference techniques for belief networks," Proc. Second European Conf. Artificial Intelligence in Medicine, pp.247-256, 1989.

[21] K.B. Hwang and B.T. Zhang, "Bayesian model averaging of Bayesian network classifiers over multiple node-orders application to sparse datasets," IEEE Trans. Syst. Man Cybern. B, vol.35, no.6, pp.1302-1310, Dec. 2005. 

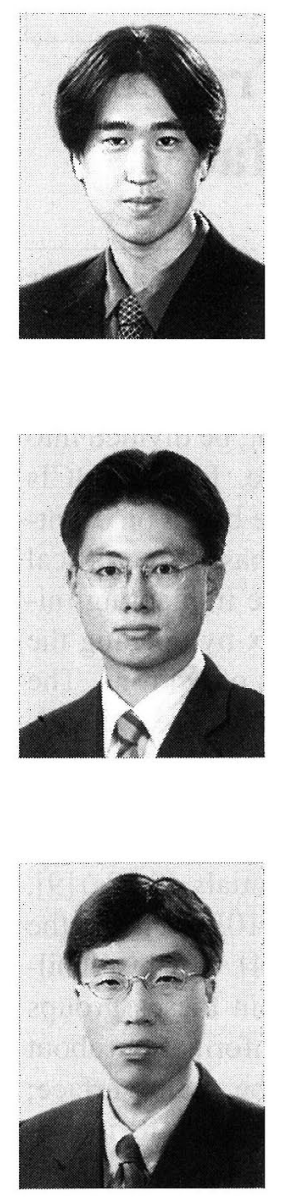

Euntai Kim was born in Seoul, Korea, in 1970. He received the B.S. (summa cum laude) and the M.S. and the Ph.D. degrees in electronic engineering, all from Yonsei University, Seoul, Korea, in 1992, 1994 and 1999, respectively. From 1999 to 2002, he was a full-time lecturer in the Department of Control and Instrumentation Engineering at Hankyong National University, Kyonggi-do, Korea. Since 2002, he has joined the faculties of the School of Electrical and Electronic Engineering at Yonsei University, where he is currently an associate professor. His current research interests include computational intelligence and its application to intelligent service robot and intelligent home network. Dr. Kim is an associate editor of International Journal of Control and Systems. 